\title{
Recurrence Relations for Moment Generating Functions of Generalized Order Statistics Based on Doubly Truncated Class of Distributions
}

\author{
A. H. Abd Ellah, ${ }^{\dagger, *}$ Abd El-Baset A. Ahmad ${ }^{\ddagger}$ \\ and Mohammad A. Fawzy ${ }^{\dagger}$ \\ † Sohag University \\ $\ddagger$ Taif University
}

\begin{abstract}
In this paper, we derived recurrence relations for joint moment generating functions of nonadjacent generalized order statistics (GOS) of random samples drawn from doubly truncated class of continuous distributions. Recurrence relations for joint moments of nonadjacent GOS (ordinary order statistics (OOS) and k-upper records (k-RVs) as special cases) are obtained. Single and product moment generating functions (moments) of nonadjacent GOS are derived. Doubly truncated new modified Weibull (Weibull, Extreme-value, exponential and Rayleigh), three Burr type XII (Lomax) and inverse Weibull distributions, among others, arise as special cases of this doubly truncated class. Two applications are introduced, the first is the characterizations for members of the class based on recurrence relations for moments of GOS, OOS and k-RVs. As the second application we found Tables of single and product moments of OOS from doubly truncated Lomax distribution.
\end{abstract}

Keywords. Generalized order statistics; recurrence relations; moment generating functions; order statistics; k-records; characterizations; truncated distributions.

* Corresponding author 


\section{Introduction}

Udo Kamps (1995) has introduced GOS as random variables having certain joint density function, which includes as a special case the joint density functions of many models of ordered random variables such as ordinary order statistics (OOS) (David, 1981; and Arnold, Balakrishnan and Nagaraja, 1992), sequential order statistics (SOS) (Cramer and Kamps, 1996), record values, k-record values (k-RVs), and Pfeifer's records (Nevzorov, 1987; and Ahsanullah, 1995), Progressive Type-II censoring order statistics (PCOS) (Balakrishnan and Asgharzadeh, 2005; and Sarhan, Ammar and Abuammoh, 2008). The structural similarities of these models are based on the similarity of their joint density function. Therefore, all of these models are contained in the model of GOS.

Our aim In this paper is derive recurrence relations for joint moment generating functions of nonadjacent GOS of random samples drawn from doubly truncated class of continuous distributions. Recurrence relations for joint moments of nonadjacent GOS (OOS and upper k-RVs as special cases) are obtained. Single and product moment generating functions (moments) of nonadjacent GOS are derived.

Recently, new and more general research based on generalized order statistics have been made by several authors. Statistical inference and characterizations based on properties generalized order statistics have been established by several authors. Such authors are Keseling (1999), Cramer and Kamps (2000), Ahsanullah (2000) Habibullah and Ahsanullah (2000), Pawlas and Szynal (2001), Ahmad and fawzy (2003), Saran and Pandey (2003), Athar and Islam (2004), AL-Hussaini et al. (2005), Ahmad (2006, 2008), Ahmad and Abu-Shal (2006), among others. A large number of publications are concerned with recurrence relations of moments of OOS and RVs, details survey of the OOS are given in Balakrishnan, Malik and Ahmed (1988), Balakrishnan and Sultan (1998), Balakrishnan Ahsanullah (1995), Ahmad (2001) and AL-Hussaini et al (2004).

Our aim in this paper is to drive recurrence relations for joint moment generating functions of nonadjacent generalized order statistics of random samples drawn from doubly truncated class of continuous distributions. Joint moments of nonadjacent GOS are obtained from these relations. Specialization to single, product moment generating functions and moments of nonadjacent GOS, OOS and RVs are obtained. Single and product moment generating functions and moments of nonadjacent GOS, OOS and RVs are 
derived as special cases of this class.

Let $X$ be a random variable (rv) has a class with distribution functions $(d f)$ which given by

$$
F(x)=1-\{a h(x)+b\}^{c}, \quad \alpha \leqslant x \leqslant \beta,
$$

and the corresponding probability density function (pdf) is given by

$$
f(x)=-\operatorname{ach}^{\prime}(x)\{a h(x)+b\}^{c-1}, \quad \alpha \leqslant x \leqslant \beta,
$$

where $a, b, c \neq 0$ are constants such that $F(\alpha)=0, F(\beta)=1$ and $h(x)$ is a monotonic differentiable arbitrary function of $x$ in $[\alpha, \beta]$ such that $f(x)$ is a $p d f$.

The corresponding survival function $(s f)$ is given by

$$
\bar{F}(x)=1-F(x)=\{a h(x)+b\}^{c}, \quad \alpha \leqslant x \leqslant \beta .
$$

so, from (2), we can obtain

$$
\bar{F}(x)=\frac{\{a h(x)+b\} f(x)}{-a c h^{\prime}(x)}
$$

The doubly truncated pdf $f_{d}(x)$, df $F_{d}(x)$, and sf $\bar{F}_{d}(x)$, are given in general forms, respectively, by

$$
\begin{gathered}
f_{d}(x)=D f(x), \quad P_{1} \leqslant x \leqslant Q_{1}, \\
F_{d}(x)=D F(x)-P_{2}, \\
\bar{F}_{d}(x)=Q_{2}+D \bar{F}(x),
\end{gathered}
$$

where

$D=\frac{1}{Q-P}, \quad Q=F\left(Q_{1}\right), \quad P=F\left(P_{1}\right), \quad Q_{2}=D(Q-1), \quad$ and $\quad P_{2}=D P$.

Substituting (3) in (4), we obtain

$$
\bar{F}_{d}(x)=Q_{2}-\frac{\{a h(x)+b\} f_{d}(x)}{a c h^{\prime}(x)} .
$$




\section{Recurrence Relation for Moment Generating Functions of GOS from Doubly Truncated Dis- tributions}

Suppose that $X_{r ; n, m, k}$ is the $r$ th GOS, so if $X_{1 ; n, m, k}, X_{2 ; n, m, k}, \ldots, X_{n ; n, m, k}$ are $n$ GOS from the $d f(5)$, where $n>1, m \geqslant-1, k \geqslant 1$ are real numbers. Then the joint doubly truncated pdf of $X_{r_{1} ; n, m, k}, \ldots, X_{r_{\ell} ; n, m, k}$ is given for $1 \leqslant r_{1} \leqslant \cdots \leqslant r_{\ell} \leqslant n, r_{0}=0, r_{\ell+1}=n+1$ (see, Ahmad and Abu-Shal, 2006), by

$$
\begin{aligned}
f_{r_{1}, \ldots, r_{\ell}}\left(x_{1}, \ldots, x_{\ell}\right)= & C\left(\ell, r_{\ell}\right)\left[\prod _ { i = 0 } ^ { \ell - 1 } \{ \overline { F } ( x _ { i } ) \} ^ { m } \left[h_{m}\left\{F\left(x_{i+1}\right)\right\}\right.\right. \\
& \left.\left.-h_{m}\left\{F\left(x_{i}\right)\right\}\right]^{r_{i+1}-r_{i}-1}\right] \times\left\{\prod_{i=1}^{\ell} f\left(x_{i}\right)\right\}\left\{\bar{F}\left(x_{l}\right)\right\}^{\gamma_{r_{l}}-1},
\end{aligned}
$$

for $F^{-1}(0+)<x_{1} \leqslant \cdots \leqslant x_{n}<F^{-1}(1)$, where

$$
C\left(\ell, r_{\ell}\right)=\frac{C_{r_{\ell}-1}}{\prod_{i=0}^{\ell-1}\left(r_{i+1}-r_{i}-1\right) !}, \quad C_{r_{\ell}-1}=\prod_{i=0}^{r_{\ell}} \gamma_{i}, \quad \gamma_{i}=k+(n-i)(m+1)
$$

and for $0<t<1$

$$
h_{m}(t)= \begin{cases}-\frac{(1-t)^{m+1}}{(m+1)} & m \neq-1 \\ -\ln (1-t) & m=-1\end{cases}
$$

The joint moment generating function of $X_{r_{1} ; n, m, k}^{j_{1}}, \ldots, X_{r_{l} ; n, m, k}^{j_{l}}$, for $j_{1}, \ldots, j_{l} \geqslant$ 1 , is given by

$$
\begin{aligned}
M_{r_{1}, \ldots, r_{\ell}, n, m, k}^{\left(j_{1}, \ldots, j_{\ell}\right)}\left(t_{1}, \ldots, t_{\ell}\right)= & E\left\{\exp \left(\sum_{i=1}^{\ell} t_{i} X_{r_{i}, n, m, k}^{j_{i}}\right)\right\} \\
= & \int_{-\infty}^{\infty} \int_{x_{1}}^{\infty} \ldots \int_{x_{\ell-1}}^{\infty} \exp \left(\sum_{i=1}^{\ell} t_{i} x_{i}^{j_{i}}\right) \\
& \times f_{r_{1}, \ldots, r_{\ell}}\left(x_{1}, \ldots, x_{\ell}\right) d x_{\ell} \ldots d x_{1},
\end{aligned}
$$

and the joint moments are given by 


$$
\begin{aligned}
\mu_{r_{1}, \ldots, r_{\ell}, n, m, k}^{\left(j_{1}, \ldots, j_{\ell}\right)}\left(t_{1}, \ldots, t_{\ell}\right) & =E\left(\prod_{i=1}^{\ell} X_{r_{i}, n, m, k}^{j_{i}}\right) \\
& =\int_{-\infty}^{\infty} \int_{x_{1}}^{\infty} \ldots \int_{x_{\ell-1}}^{\infty} \prod_{i=1}^{\ell} x_{i}^{j_{i}} f_{r_{1}, \ldots, r_{\ell}}\left(x_{1}, \ldots, x_{\ell}\right) d x_{\ell} \ldots d x_{1} .
\end{aligned}
$$

Now we can a recurrence relation for joint moment generating functions of nonadjacent GOS for the class (5) by the following theorem.

Theorem 1. Let $X_{r_{1} ; n, m, k}, \ldots, X_{r_{\ell} ; n, m, k}$ be $n$ GOS formed from a random sample of size $n$ drawn form the $d f(5)$. Suppose for $j_{1}, \ldots, j_{\ell} \geqslant 1,1 \leqslant r_{1} \leqslant$ $\cdots \leqslant r_{\ell} \leqslant n$, that the expectation

$$
E\left\{X_{r_{\ell}, n, m, k}^{j_{\ell}-1} \exp \left(\sum_{i=1}^{\ell} t_{i} X_{r_{i}, n, m, k}^{j_{i}}\right) \cdot \frac{a h\left(X_{r_{\ell}, n, m, k}\right)+b}{h^{\prime}\left(X_{r_{\ell}, n, m, k}\right)}\right\},
$$

is finite, then for $r_{\ell-1}<r_{\ell}^{*}<r_{\ell}, m \geqslant-1$, and $k \geqslant 1$ the following recurrence relation

$$
\begin{aligned}
& M_{r_{1}, \ldots, r_{\ell}, n, m, k}^{\left(j_{1}, \ldots, j_{\ell}\right)}\left(t_{1}, \ldots, t_{\ell}\right)-M_{r_{1}, \ldots, r_{\ell}^{*}, n, m, k}^{\left(j_{1}, \ldots, j_{j}\right)}\left(t_{1}, \ldots, t_{\ell}\right)= \\
& \xi Q_{2}\left\{M_{r_{1}, \ldots, r_{\ell}, n-1, m, k+m}^{\left(j_{1}, \ldots, j_{\ell}\right)}\left(t_{1}, \ldots, t_{\ell}\right)-M_{r_{1}, \ldots, r_{\ell}^{*}, n-1, m, k+m}^{\left(j_{1}, \ldots, j_{\ell}\right)}\left(t_{1}, \ldots, t_{\ell}\right)\right\} \\
& -\frac{t_{\ell} j_{\ell}}{a c \gamma_{r_{\ell}}} E\left\{\Psi_{j_{\ell}}\left(X_{r_{\ell}, n, m, k} ; t_{\ell}\right) \exp \left(\sum_{i=1}^{\ell-1} t_{i} X_{r_{i}, n, m, k}^{j_{i}}\right)\right\}
\end{aligned}
$$

where

$$
\begin{gathered}
\Psi_{j_{\ell}}\left(x_{r_{\ell}, n, m, k} ; t_{\ell}\right)=x_{r_{\ell}, n, m, k}^{j_{\ell}-1} \exp \left(t_{\ell} x_{r_{\ell}, n, m, k}^{j_{\ell}}\right) \cdot \frac{a h\left(x_{r_{\ell}, n, m, k}\right)+b}{h^{\prime}\left(x_{r_{\ell}, n, m, k}\right)} \\
\xi=\xi\left(r_{\ell}, n, m, k\right)=\prod_{i=1}^{r_{\ell}-1} \frac{\gamma_{i}}{\gamma_{i}^{*}}, \quad \gamma_{i}^{*}=\gamma_{i}-1=(k+m)+(n-1-i)(m+1) \\
\text { and } \quad \gamma_{r}+m=\gamma_{r-1}-1 .
\end{gathered}
$$


Proof. By replacing $f$ and $\bar{F}$ in $(6)$ by $f_{d}$ and $\overline{F_{d}}$ respectively, we can write (8) as

$$
\begin{aligned}
M_{r_{1}, \ldots, r_{\ell}, n, m, k}^{\left(j_{1}, \ldots, j_{\ell}\right)}\left(t_{1}, \ldots, t_{\ell}\right)= & C\left(\ell, r_{\ell}\right) \int_{P_{1}}^{Q_{1}} \int_{x_{1}}^{Q_{1}} \ldots \int_{x_{\ell-1}}^{Q_{1}} \exp \left(\sum_{i=1}^{\ell} t_{i} x_{i}^{j_{i}}\right) \\
& \times\left\{\prod_{i=1}^{\ell} f_{d}\left(x_{i}\right)\right\} \cdot\left[\prod _ { i = 0 } ^ { \ell - 1 } \{ \overline { F } _ { d } ( x _ { i } ) \} ^ { m } \left[h_{m}\left\{F_{d}\left(x_{i+1}\right)\right\}\right.\right. \\
& \left.\left.-h_{m}\left\{F_{d}\left(x_{i}\right)\right\}\right]^{r_{i+1}-r_{i}-1}\right]\left\{\bar{F}_{d}\left(x_{\ell}\right)\right\}^{\gamma_{r_{\ell}}-1} d x_{\ell} \ldots d x_{1} \\
= & C\left(\ell, r_{\ell}\right) \int_{P_{1}}^{Q_{1}} \int_{x_{1}}^{Q_{1}} \ldots \int_{x_{\ell-1}}^{Q_{1}} \exp \left(\sum_{i=1}^{\ell} t_{i} x_{i}^{j_{i}}\right) \\
& \times\left\{\prod_{i=1}^{\ell-1} f_{d}\left(x_{i}\right)\right\} \cdot\left[\prod _ { i = 0 } ^ { \ell - 2 } \{ \overline { F } _ { d } ( x _ { i } ) \} ^ { m } \left[h_{m}\left\{F_{d}\left(x_{i+1}\right)\right\}\right.\right. \\
& \left.-h_{m}\left\{F_{d}\left(x_{i}\right)\right\}\right]^{r_{i+1}-r_{i}-1}\left\{\bar{F}_{d}\left(x_{\ell}\right)\right\}^{m} \\
& \times I\left(x_{\ell-1}\right) d x_{\ell-1} \ldots d x_{1},
\end{aligned}
$$

where

$$
\begin{aligned}
I\left(x_{\ell-1}\right)= & \int_{x_{\ell-1}}^{Q_{1}} \exp \left(t_{\ell} x_{\ell}^{j_{\ell}}\right)\left[h_{m}\left\{F_{d}\left(x_{\ell}\right)\right\}-h_{m}\left\{F_{d}\left(x_{\ell-1}\right)\right\}\right]^{r_{\ell}-r_{\ell-1}-1} \\
& \times\left\{\bar{F}_{d}\left(x_{\ell}\right)\right\}^{\gamma_{r_{\ell}}-1}\left\{f_{d}\left(x_{\ell}\right)\right\} d x_{\ell} \\
= & \int_{x_{\ell-1}}^{Q_{1}} \exp \left(t_{\ell} x_{\ell}^{j_{\ell}}\right)\left[h_{m}\left\{F_{d}\left(x_{\ell}\right)\right\}-h_{m}\left\{F_{d}\left(x_{\ell-1}\right)\right\}\right]^{r_{\ell}-r_{\ell-1}-1} \\
& d\left\{-\bar{F}_{d}\left(x_{\ell}\right)\right\}^{\frac{\gamma_{r_{\ell}}}{r_{\ell}}}
\end{aligned}
$$

making use of integration by parts we can write $I\left(x_{\ell-1}\right)$ in the form

$$
\begin{aligned}
I\left(x_{\ell-1}\right)= & \frac{t_{\ell} j_{\ell}}{a c \gamma_{r_{\ell}}} \int_{x_{\ell-1}}^{Q_{1}} x_{\ell}^{j_{\ell-1}} \exp \left(t_{\ell} x_{\ell}^{j_{\ell}}\right)\left[h_{m}\left\{F_{d}\left(x_{\ell}\right)\right\}-h_{m}\left\{F_{d}\left(x_{\ell-1}\right)\right\}\right]^{r_{\ell}-r_{\ell-1}-1} \\
& \times\left\{\bar{F}_{d}\left(x_{\ell}\right)\right\}^{\gamma_{\ell}} d x_{\ell}+\frac{r_{\ell}-r_{\ell-1}-1}{\gamma_{r_{\ell}}} \int_{x_{\ell-1}}^{Q_{1}} \exp \left(t_{\ell} x_{\ell}^{j_{\ell}}\right)\left[h_{m}\left\{F_{d}\left(x_{\ell}\right)\right\}\right.
\end{aligned}
$$




$$
\left.-h_{m}\left\{F_{d}\left(x_{\ell-1}\right)\right\}\right]^{r_{\ell}-r_{\ell-1}-2}\left\{\bar{F}_{d}\left(x_{\ell}\right)\right\}^{\gamma_{r_{\ell-1}}-1}\left\{f_{d}\left(x_{\ell}\right)\right\} d x_{\ell},
$$

substituting $I\left(x_{\ell-1}\right)$ in (11), we obtain

$$
\begin{aligned}
M_{r_{1}, \ldots, r_{\ell}, n, m, k}^{\left(j_{1}, \ldots, j_{\ell}\right)} & \left(t_{1}, \ldots, t_{\ell}\right)=\frac{t_{\ell} j_{\ell} C\left(\ell, r_{\ell}\right)}{\gamma_{r_{\ell}}} \int_{P_{1}}^{Q_{1}} \int_{x_{1}}^{Q_{1}} \ldots \int_{x_{\ell-1}}^{Q_{1}} x_{\ell}^{j_{\ell}-1} \exp \left(\sum_{i=1}^{\ell} t_{i} x_{i}^{j_{i}}\right) \\
& \times\left[\prod_{i=0}^{\ell-1}\left\{\bar{F}_{d}\left(x_{i}\right)\right\}^{m}\left[h_{m}\left\{F_{d}\left(x_{i+1}\right)\right\}-h_{m}\left\{F_{d}\left(x_{i}\right)\right\}\right]^{r_{i+1}-r_{i}-1}\right] \\
& \times\left\{\bar{F}_{d}\left(x_{\ell}\right)\right\}^{\gamma_{\ell}}\left\{\prod_{i=1}^{\ell-1} f_{d}\left(x_{i}\right)\right\} d x_{\ell} \ldots d x_{1}+\frac{\left(r_{\ell}-r_{\ell-1}-1\right) C\left(\ell, r_{\ell}\right)}{\gamma_{r_{\ell}}} \\
& \times \int_{P_{1}}^{Q_{1}} \int_{x_{1}}^{Q_{1}} \ldots \int_{x_{\ell-1}}^{Q_{1}} \exp \left(\sum_{i=1}^{\ell} t_{i} x_{i}^{j_{i}}\right)\left\{\prod_{i=0}^{\ell} f_{d}\left(x_{i}\right)\right\} \\
& \times\left\{\bar{F}_{d}\left(x_{\ell}\right)\right\}^{\gamma_{r_{\ell-1}}-1}\left[\prod _ { i = 0 } ^ { \ell - 1 } \{ \overline { F } _ { d } ( x _ { i } ) \} ^ { m } \left[h_{m}\left\{F_{d}\left(x_{i+1}\right)\right\}\right.\right. \\
& \left.-h_{m}\left\{F_{d}\left(x_{i}\right)\right\}\right]^{r_{i+1}-r_{i}-1} d x_{\ell} \ldots d x_{1} .
\end{aligned}
$$

from (7) we can show that

$$
\frac{\left(r_{\ell}-r_{\ell-1}-1\right) C\left(\ell, r_{\ell}\right)}{\gamma_{r_{\ell}}}=\frac{C_{r_{\ell}^{*}-1}}{\prod_{i=1}^{\ell-2}\left(r_{i}-r_{i-1}-1\right) !\left(r_{\ell}^{*}-r_{\ell-1}-1\right)}
$$

where

$$
C^{*}\left(\ell, r_{\ell}\right)=\frac{\prod_{i=0}^{r_{\ell}} \gamma_{i}^{*}}{\prod_{i=0}^{\ell-1}\left(r_{i+1}-r_{i}-1\right) !}, \quad \gamma_{i}^{*}=\gamma_{i}-1 .
$$

so (12) can be written as

$$
\begin{aligned}
& M_{r_{1}, \ldots, r_{\ell}, n, m, k}^{\left(j_{1}, \ldots, j_{\ell}\right)}\left(t_{1}, \ldots, t_{\ell}\right)-M_{r_{1}, \ldots, r_{\ell}^{*}, n, m, k}^{\left(j_{1}, \ldots, j_{\ell}\right)}\left(t_{1}, \ldots, t_{\ell}\right)=\frac{t_{\ell} j_{\ell} C\left(\ell, r_{\ell}\right)}{\gamma_{r_{\ell}}} \\
& \times \int_{P_{1}}^{Q_{1}} \int_{x_{1}}^{Q_{1}} \ldots \int_{x_{\ell-1}}^{Q_{1}} x_{\ell}^{j_{\ell-1}} \exp \left(\sum_{i=1}^{\ell} t_{i} x_{i}^{j_{i}}\right)\left\{\prod_{i=1}^{\ell-1} f_{d}\left(x_{i}\right)\right\}\left\{\bar{F}_{d}\left(x_{\ell}\right)\right\}^{\gamma_{r_{\ell}}}
\end{aligned}
$$




$$
\times\left[\prod_{i=0}^{\ell-1}\left\{\bar{F}_{d}\left(x_{i}\right)\right\}^{m}\left[h_{m}\left\{F_{d}\left(x_{i+1}\right)\right\}-h_{m}\left\{F_{d}\left(x_{i}\right)\right\}\right]^{r_{i+1}-r_{i}-1}\right] d x_{\ell} \ldots d x_{1},
$$

Making use of (5) we can write (13) in the form

$$
\begin{aligned}
& M_{r_{1}, \ldots, r_{\ell}, n, m, k}^{\left(j_{1}, \ldots, j_{\ell}\right)}\left(t_{1}, \ldots, t_{\ell}\right)-M_{r_{1}, \ldots, r_{\ell}^{*}, n, m, k}^{\left(j_{1}, \ldots, j_{\ell}\right)}\left(t_{1}, \ldots, t_{\ell}\right)=\frac{t_{\ell} j_{\ell} Q_{2} C\left(\ell, r_{\ell}\right)}{\gamma_{r_{\ell}}} \\
& \times \int_{P_{1}}^{Q_{1}} \int_{x_{1}}^{Q_{1}} \ldots \int_{x_{\ell-1}}^{Q_{1}} x_{\ell}^{j_{\ell}-1} \exp \left(\sum_{i=1}^{\ell} t_{i} x_{i}^{j_{i}}\right)\left\{\prod_{i=1}^{\ell-1} f_{d}\left(x_{i}\right)\right\} \\
& \times\left[\prod_{i=0}^{\ell-1}\left\{\bar{F}_{d}\left(x_{i}\right)\right\}^{m}\left[h_{m}\left\{F_{d}\left(x_{i+1}\right)\right\}-h_{m}\left\{F_{d}\left(x_{i}\right)\right\}\right]^{r_{i+1}-r_{i}-1}\right] \\
& \times\left\{\bar{F}_{d}\left(x_{\ell}\right)\right\}^{\gamma_{r_{\ell}}-1} d x_{\ell} \ldots d x_{1}-\frac{t_{\ell} j_{\ell} C\left(\ell, r_{\ell}\right)}{a c \gamma_{r_{\ell}}} \\
& \times \int_{P_{1}}^{Q_{1}} \int_{x_{\ell}}^{Q_{1}} \ldots \int_{x_{\ell-1}}^{Q_{1}} x_{\ell}^{j_{\ell}-1} \exp \left(\sum_{i=1}^{\ell} t_{i} x_{i}^{j_{i}}\right) \frac{a h\left(x_{\ell}\right)+b}{h^{\prime}\left(x_{\ell}\right)} \\
& \times\left\{\prod_{i=1}^{\ell} f_{d}\left(x_{i}\right)\right\}\left\{\bar{F}_{d}\left(x_{\ell}\right)\right\}^{\gamma_{r_{\ell}}-1}\left[\prod_{i=0}^{\ell-1}\left\{\bar{F}_{d}\left(x_{i}\right)\right\}^{m}\right]\left[h_{m}\left\{F_{d}\left(x_{i+1}\right)\right\}\right. \\
&\left.\left.-h_{m}\left\{F_{d}\left(x_{i}\right)\right\}\right]^{r_{i+1}-r_{i}-1}\right] d x_{\ell} \ldots d x_{1},
\end{aligned}
$$

from (8) in the last term we can write (14) in the form

$$
\begin{aligned}
M_{r_{1}, \ldots, r_{\ell}, n, m, k}^{\left(j_{1}, \ldots, j_{\ell}\right)}\left(t_{1}, \ldots, t_{\ell}\right)-M_{r_{1}, \ldots, r_{\ell}}^{\left(j_{1}, \ldots, j_{\ell}, m, k\right.} & \left(t_{1}, \ldots, t_{\ell}\right)=\frac{t_{\ell} j_{\ell} Q_{2} C\left(\ell, r_{\ell}\right)}{\gamma_{r_{\ell}}} \\
& \times \int_{P_{1}}^{Q_{1}} \int_{x_{1}}^{Q_{1}} \ldots \int_{x_{\ell-1}}^{Q_{1}} x_{\ell}^{j_{\ell}-1} \exp \left(\sum_{i=1}^{\ell} t_{i} x_{i}^{j_{i}}\right)\left\{\prod_{i=1}^{\ell-1} f_{d}\left(x_{i}\right)\right\} \\
& \times\left[\prod_{i=0}^{\ell-1}\left\{\bar{F}_{d}\left(x_{i}\right)\right\}^{m}\left[h_{m}\left\{F_{d}\left(x_{i+1}\right)\right\}-h_{m}\left\{F_{d}\left(x_{i}\right)\right\}\right]^{r_{i+1}-r_{i}-1}\right] \\
& \times\left\{\bar{F}_{d}\left(x_{\ell}\right)\right\}^{\gamma_{r_{\ell}}-1} d x_{\ell} \ldots d x_{1}-\frac{t_{\ell} j_{\ell}}{a c \gamma_{r_{\ell}}} E\left[X_{r_{\ell}, n, m, k}^{j_{\ell}-1}\right.
\end{aligned}
$$




$$
\left.\times \exp \left(\sum_{i=1}^{\ell} t_{i} X_{r_{i}, n, m, k}^{j_{i}}\right) \frac{a h\left(X_{r_{\ell}, n, m, k}\right)+b}{h^{\prime}\left(X_{r_{\ell}, n, m, k}\right)}\right],
$$

then by replacing $n$ by $n-1$ and $k$ by $k+m$ in (13), and substituting in the first term in the right hand side in (15), the recurrence relation (9) can be obtained.

Theorem 2. The recurrence relation (9) is satisfied, if and only if X has the $d f(5)$.

Proof. If X has $d f$ (5), then recurrence relation (9) is satisfied from Theorem 1. On the other hand, if the recurrence relation (9) is satisfied, then by using (6) and (13) in (9), we obtain

$$
\begin{aligned}
\int_{P_{1}}^{Q_{1}} \int_{x_{1}}^{Q_{1}} \ldots \int_{x_{\ell-1}}^{Q_{1}} x_{\ell}^{j_{\ell-1}} \exp \left(\sum_{i=1}^{\ell} t_{i} x_{i}^{j_{i}}\right)\left[\prod _ { i = 0 } ^ { \ell - 1 } \{ \overline { F } _ { d } ( x _ { i } ) \} ^ { m } \left[h_{m}\left\{F_{d}\left(x_{i+1}\right)\right\}\right.\right. \\
\left.\left.-h_{m}\left\{F_{d}\left(x_{i}\right)\right\}\right]^{r_{i+1}-r_{i}-1}\right] \cdot\left\{\prod_{i=1}^{\ell-1} f_{d}\left(x_{i}\right)\right\}\left\{\bar{F}_{d}\left(x_{\ell}\right)\right\}^{\gamma_{r_{\ell}}-1} \\
\\
{\left[\bar{F}_{d}\left(x_{\ell}\right)-Q_{2}+\frac{\left\{a h\left(x_{\ell}\right)+b\right\}}{a c h^{\prime}\left(x_{\ell}\right)} f_{d}\left(x_{\ell}\right)\right] d x_{\ell} \ldots d x_{1}=0 . }
\end{aligned}
$$

Applying the extension of Müntz-Szàsz theorem, see Hwang and Lin (1984), we have

$$
\bar{F}_{d}\left(x_{\ell}\right)-Q_{2}+\frac{\left\{a h\left(x_{\ell}\right)+b\right\}}{a c h^{\prime}\left(x_{\ell}\right)} f_{d}\left(x_{\ell}\right)=0
$$

This leads to

$$
\bar{F}_{d}\left(x_{\ell}\right)=Q_{2}-\frac{\left\{a h\left(x_{\ell}\right)+b\right\}}{a c h^{\prime}\left(x_{\ell}\right)} f_{d}\left(x_{\ell}\right) .
$$

whose solution is the $d f(5)$.

\subsection{Remarks}

1. If $r_{\ell}=r_{\ell-1}+1$, then

$$
I\left(x_{\ell-1}\right)=\int_{x_{\ell-1}}^{Q_{1}} \exp \left(t_{\ell} x_{\ell}^{j_{\ell}}\right)\left\{\bar{F}_{d}\left(x_{\ell}\right)\right\}^{\gamma_{r_{\ell}}-1}\left\{f_{d}\left(x_{\ell}\right)\right\} d x_{\ell},
$$


by substituting $I\left(x_{\ell-1}\right)$ in (9), and by the same way in the above proof we can obtain

$$
\begin{array}{r}
M_{r_{1}, \ldots, r_{\ell}, n, m, k}^{\left(j_{1}, \ldots, j_{\ell}\right)}\left(t_{1}, \ldots, t_{\ell}\right)=\xi Q_{2} M_{r_{1}, \ldots, r_{\ell}, n-1, m, k+m}^{\left(j_{1}, \ldots, j_{\ell}\right)}\left(t_{1}, \ldots, t_{\ell}\right)-\frac{t_{\ell} j_{\ell}}{a c \gamma_{r_{\ell}}} \\
\times E\left\{\Psi_{j_{\ell}}\left(x_{r_{\ell}, n, m, k} ; t_{\ell}\right) \exp \left(\sum_{i=1}^{\ell-1} t_{i} X_{r_{i}, n, m, k}^{j_{i}}\right)\right\},
\end{array}
$$

2. By differentiating (9), with respect to $t_{1}, \ldots, t_{\ell}$ and putting $t_{1}=\cdots=$ $t_{\ell}=0$, we obtain the following recurrence relation for the joint moments of nonadjacent GOS

$$
\begin{gathered}
\mu_{r_{1}, \ldots, r_{\ell}, n, m, k}^{\left(j_{1}, \ldots, j_{\ell}\right)}-\mu_{r_{1}, \ldots, r_{\ell}^{*}, n, m, k}^{\left(j_{1}, \ldots, j_{\ell}\right)}=-\frac{j_{\ell}}{a c \gamma_{r_{\ell}}} E\left\{\Psi _ { j _ { \ell } , \ldots , j _ { 1 } } \left(X_{r_{\ell}, n, m, k}, \ldots,\right.\right. \\
\left.\left.X_{r_{1}, n, m, k}\right)\right\}+\xi Q_{2} \Phi_{r_{1}, \ldots, r_{\ell}, n-1, m, k+m}^{\left(j_{1}, \ldots, j_{\ell}\right)}
\end{gathered}
$$

where

$$
\Phi_{r_{1}, \ldots, r_{\ell}, n-1, m, k+m}^{\left(j_{1}, \ldots, j_{\ell}\right)}=\mu_{r_{1}, \ldots, r_{\ell}, n-1, m, k+m}^{\left(j_{1}, \ldots, j_{\ell}\right)}-\mu_{r_{1}, \ldots, r_{\ell}^{*}, n-1, m, k+m}^{\left(j_{1}, \ldots, j_{\ell}\right)},
$$

and

$\Psi_{j_{\ell}, \ldots, j_{1}}\left(x_{r_{\ell}, n, m, k}, \ldots, x_{r_{1}, n, m, k}\right)=x_{r_{\ell}, n, m, k}^{j_{\ell}-1}\left(\prod_{i=1}^{\ell-1} x_{r_{i}, n, m, k}^{j_{i}}\right) \frac{a h\left(x_{r_{\ell}, n, m, k}\right)+b}{h^{\prime}\left(x_{r_{\ell}, n, m, k}\right)}$.

3. In the case of OOS $\left[m=0, k=1, \gamma_{i}=n-i+1\right.$ and $\left.\xi=\frac{n}{n-r_{\ell}+1}\right]$, relation (9) reduces to

$$
\begin{aligned}
M_{r_{1}, \ldots, r_{\ell}: n}^{\left(j_{1}, \ldots, j_{\ell}\right)}( & \left.t_{1}, \ldots, t_{\ell}\right)-M_{r_{1}, \ldots, r_{\ell}^{*}: n}^{\left(j_{1}, \ldots, j_{l}\right)}\left(t_{1}, \ldots, t_{\ell}\right)=\frac{n Q_{2}}{n-r_{\ell}+1} \\
& \times\left\{M_{r_{1}, \ldots, r_{\ell}: n-1}^{\left(j_{1}, \ldots, j_{\ell}\right)}\left(t_{1}, \ldots, t_{\ell}\right)-M_{r_{1}, \ldots, r_{\ell}^{*}: n-1}^{\left(j_{1}, \ldots, j_{\ell}\right)}\left(t_{1}, \ldots, t_{\ell}\right)\right\} \\
& -\frac{t_{\ell} j_{\ell}}{a c\left(n-r_{\ell}+1\right)} E\left\{\Psi_{j_{\ell}}\left(X_{r_{\ell}: n} ; t_{\ell}\right) \exp \left(\sum_{i=1}^{\ell-1} t_{i} X_{r_{i}: n}^{j_{i}}\right)\right\},
\end{aligned}
$$


and relation (17) reduces to

$$
\begin{aligned}
\mu_{r_{1}, \ldots, r_{\ell}: n}^{\left(j_{1}, \ldots, j_{\ell}\right)}-\mu_{r_{1}, \ldots, r_{\ell}^{*}: n}^{\left(j_{1}, \ldots, j_{\ell}\right)}= & -\frac{j_{\ell}}{a c\left(n-r_{\ell}+1\right)} E\left\{\Psi_{j_{\ell}, \ldots, j_{1}}\left(X_{r_{\ell}: n}, \ldots, x_{r_{1}: n}\right)\right\} \\
& +\frac{n Q_{2}}{n-r_{\ell}+1} \Phi_{r_{1}, \ldots, r_{\ell}, n-1}^{\left(j_{1}, \ldots, j_{\ell}\right)}
\end{aligned}
$$

where

$$
X_{r_{1}, \ldots, r_{\ell} ; n, 0,1} \equiv X_{r_{1}, \ldots, r_{\ell}: n} .
$$

(The recurrence relation (21) agrees with AL-Hussaini et al. (2004) when $h(x)=e^{-\lambda(x)}, a=1, b=0$ and $\left.c=1\right)$.

4. In the case of $k r v\left[m=-1, k \geqslant 1, \gamma_{i}=k\right.$ and $\left.\left(1-k^{-1}\right)^{1-r_{\ell}}\right]$, relation (9) reduces to

$$
\begin{aligned}
M_{\left(r_{1}, \ldots, r_{\ell} ; k\right)}^{\left(j_{1}, \ldots, j_{\ell}\right)}( & \left.t_{1}, \ldots, t_{\ell}\right)-M_{\left(r_{1}, \ldots, r_{\ell}^{*} ; k\right)}^{\left(j_{1}, \ldots, j_{\ell}\right)}\left(t_{1}, \ldots, t_{\ell}\right)=Q_{2}\left(1-k^{-1}\right)^{1-r_{\ell}} \\
& \times\left\{M_{\left(r_{1}, \ldots, r_{\ell} ; k-1\right)}^{\left(j_{1}, \ldots, j_{\ell}\right)}\left(t_{1}, \ldots, t_{\ell}\right)-M_{\left(r_{1}, \ldots, r_{\ell}^{*} ; k-1\right)}^{\left(j_{1}, \ldots, j_{\ell}\right)}\left(t_{1}, \ldots, t_{\ell}\right)\right\} \\
& -\frac{t_{\ell} j_{\ell}}{a c k} E\left\{\Psi_{j_{\ell}}\left(X_{\left(r_{\ell}, k\right)} ; t_{\ell}\right) \exp \left(\sum_{i=1}^{\ell-1} t_{i} X_{\left(r_{i}, k\right)}^{j_{i}}\right)\right\},
\end{aligned}
$$

and relation (17) reduces to

$$
\begin{aligned}
\mu_{\left(r_{1}, \ldots, r_{\ell}, k\right)}^{\left(j_{1}, \ldots, j_{\ell}\right)}-\mu_{\left(r_{1}, \ldots, r_{\ell}^{*} ; k\right)}^{\left(j_{1}, \ldots, j_{\ell}\right)}= & -\frac{j_{\ell}}{a c k} E\left[\Psi_{j_{\ell}, \ldots, j_{1}}\left\{X_{\left(r_{\ell}, k\right)}, \ldots, X_{\left(r_{1}, k\right)}\right\}\right] \\
& +Q_{2}\left(1-k^{-1}\right)^{1-r_{\ell}} \Phi_{\left(r_{1}, \ldots, r_{\ell}, k-1\right)}^{\left(j_{1}, \ldots, j_{\ell}\right)}
\end{aligned}
$$

where

$$
X_{r_{1}, \ldots, r_{\ell}, n,-1, k} \equiv X_{\left(r_{1}, \ldots, r_{\ell}, k\right)} .
$$

The recurrence relations for RVs was obtain if we put $k=1$ in (23).

5. If we put $j_{1}=\cdots=j_{\ell-1}=0, j_{\ell}=j, r_{\ell}=r, t_{1}=\cdots=t_{\ell-1}=0$ and $t_{\ell}=t$, in (9) and (15), respectively, we have

$$
\begin{aligned}
M_{r, n, m, k}^{(j)}(t)-M_{r-1, n, m, k}^{(j)}(t)= & \xi Q_{2}\left\{M_{r, n-1, m, k+m}^{(j)}(t)-M_{r-1, n-1, m, k+m}^{(j)}(t)\right\} \\
& -\frac{t j}{a c \gamma_{r}} E\left\{\Psi_{j}\left(X_{r, n, m, k} ; t\right)\right\}
\end{aligned}
$$


and

$$
\mu_{r, n, m, k}^{(j)}-\mu_{r-1, n, m, k}^{(j)}=-\frac{j}{a c \gamma_{r}} E\left\{\Psi_{j}\left(X_{r, n, m, k}\right)\right\}+\xi Q_{2} \Phi_{r, n-1, m, k+m}^{(j)} .
$$

(Recurrence relation (25) agrees with (2.5) of Mahmoud and Al-Nagar (2006) if $\left.\phi(x)=x^{j}\right)$

and when $h(x)=\exp \{-\lambda(x)\}, a=1, b=0$ and $c=1$, this result agrees with one of Ahmad and Fawzy (2003) results.

6. If we put $j_{1}=\cdots=j_{\ell-2}=0, j_{\ell-1}=e, j_{\ell}=\varepsilon, r_{\ell-1}=r, r_{\ell}=s$ $t_{1}=\cdots=t_{\ell-2}=0 t_{\ell-1}=t_{1}$ and $t_{\ell}=t_{2}$, in (9) and (17), we obtain

$$
\begin{gathered}
M_{r, s, n, m, k}^{(e, \varepsilon)}\left(t_{\left.1, t_{2}\right)-} M_{r, s-1, n, m, k}^{(e, \varepsilon)}\left(t_{1}, t_{2}\right)=\xi Q_{2}\left\{M_{r, s, n-1, m, k+m}^{(e, \varepsilon)}\left(t_{1, t_{2}}\right)\right.\right. \\
\left.-M_{r, s-1, n-1, m, k+m}^{(e, \varepsilon)}\left(t_{\left.1, t_{2}\right)}\right)\right\}-\frac{t_{2} \varepsilon}{a c \gamma_{s}} \\
\times E\left\{\Psi_{\varepsilon}\left(X_{s, n, m, k} ; t_{2}\right) \exp \left(t_{1} X_{r, n, m, k}^{e}\right)\right\} \\
\mu_{r, s, n, m, k}^{(e, \varepsilon)}-\mu_{r, s-1, n, m, k}^{(e, \varepsilon)}=-\frac{\varepsilon}{a c \gamma_{s}} E\left\{\Psi_{e, \varepsilon}\left(X_{s, n, m, k}, X_{r, n, m, k}\right)\right\} \\
+\xi Q_{2} \Phi_{r, s, n-1, m, k+m}^{(e, \varepsilon)}
\end{gathered}
$$

Recurrence relation (27) agrees with (3.4) of Mahmoud and Al-Nagar (2006) if $\phi\left(x_{r} x_{s}\right)=x_{r}^{e} x_{s}^{\varepsilon}$.

7. In the case OOS, relations (25) and (27), reduce to

$$
\mu_{r: n}^{(j)}-\mu_{r-1: n}^{(j)}=-\frac{j}{a c(n-r+1)} E\left\{\Psi_{j}\left(X_{r: n}\right)\right\}+\frac{n Q_{2}}{(n-r+1)} \Phi_{r: n-1}^{(j)},
$$

and

$$
\begin{aligned}
\mu_{r, s: n}^{(e, \varepsilon)}-\mu_{r, s-1: n}^{(e, \varepsilon)}= & -\frac{\varepsilon}{a c(n-s+1)} E\left\{\Psi_{e, \varepsilon}\left(X_{s: n}, X_{r: n}\right)\right\} \\
& +\frac{n Q_{2}}{n-s+1} \Phi_{r, s: n-1}^{(e, \varepsilon)} .
\end{aligned}
$$


8. In the case of k-RVs (25) and (27), reduce to

$$
\mu_{(r, k)}^{(j)}-\mu_{(r-1, k)}^{(j)}=-\frac{j}{a c k} E\left\{\Psi_{j}\left(X_{(r, k)}\right)\right\}+Q_{2}\left(1-k^{-1}\right)^{1-r} \Phi_{(r, k-1)}^{(j)},
$$

and

$$
\begin{aligned}
\mu_{(r, s, k)}^{(e, \varepsilon)}-\mu_{(r, s-1, k)}^{(e, \varepsilon)}= & -\frac{\varepsilon}{a c k} E\left\{\Psi_{e, \varepsilon}\left(X_{(s, k)}, X_{(r, k)}\right)\right\} \\
& +Q_{2}\left(1-k^{-1}\right)^{1-r} \Phi_{(r, s, k-1)}^{(e, \varepsilon)} .
\end{aligned}
$$

\subsection{Special Cases}

1) The left-truncated case can be obtained when $Q_{2}=0$, so relations (9) and (17), reduce to

$$
\begin{gathered}
M_{r_{1}, \ldots, r_{\ell}, n, m, k}^{\left(j_{1}, \ldots, j_{\ell}\right)}\left(t_{1}, \ldots, t_{\ell}\right)-M_{r_{1}, \ldots, r_{\ell}, n, m, k}^{\left(j_{1}, \ldots, j_{\ell}\right)}\left(t_{1}, \ldots, t_{\ell}\right)=-\frac{t_{\ell} j_{\ell}}{a c \gamma_{r_{\ell}}} \\
E\left\{\Psi_{j_{\ell}}\left(x_{r_{\ell}, n, m, k} ; t_{\ell}\right) \exp \left(\sum_{i=1}^{\ell-1} t_{i} X_{r_{i}, n, m, k}^{j_{i}}\right)\right\},
\end{gathered}
$$

and

$$
\begin{gathered}
\mu_{r_{1}, \ldots, r_{\ell}, n, m, k}^{\left(j_{1}, \ldots, j_{\ell}\right)}-\mu_{r_{\ell}, \ldots, r_{\ell}^{*}, n, m, k}^{\left(j_{1}, \ldots, j_{\ell}\right)}=-\frac{j_{\ell}}{a c \gamma_{r_{\ell}}} E\left\{\Psi _ { j _ { \ell } , \ldots , j _ { 1 } } \left(X_{r_{\ell}, n, m, k}, \ldots,\right.\right. \\
\left.\left.X_{r_{1}, n, m, k}\right)\right\} .
\end{gathered}
$$

2) The right-truncated case can be obtained $Q_{2}=\frac{Q-1}{Q}$, so relations (9) and (17), reduce to

$$
\begin{aligned}
& M_{r_{1}, \ldots, r_{\ell}, n, m, k}^{\left(j_{1}, \ldots, j_{\ell}\right)}\left(t_{1}, \ldots, t_{\ell}\right)-M_{r_{1}, \ldots, r_{\ell}^{*}, n, m, k}^{\left(j_{1}, \ldots, j_{\ell}\right)}\left(t_{1}, \ldots, t_{\ell}\right)=\xi \frac{Q-1}{Q} \\
& \quad\left\{M_{r_{1}, \ldots, r_{\ell}, n-1, m, k+m}^{\left(j_{1}, \ldots, j_{\ell}\right)}\left(t_{1}, \ldots, t_{\ell}\right)-M_{r_{1}, \ldots, r_{\ell}^{*}, n-1, m, k+m}^{\left(j_{1}, \ldots, j_{\ell}\right)}\left(t_{1}, \ldots, t_{\ell}\right)\right\} \\
& \quad-\frac{t_{\ell} j_{\ell}}{a c \gamma_{r_{\ell}}} E\left\{\Psi_{j_{\ell}}\left(X_{r_{\ell}, n, m, k} ; t_{\ell}\right) \exp \left(\sum_{i=1}^{\ell-1} t_{i} X_{r_{i}, n, m, k}^{j_{i}}\right)\right\},
\end{aligned}
$$

and

$$
\begin{aligned}
\mu_{r_{1}, \ldots, r_{\ell}, n, m, k}^{\left(j_{1}, \ldots, j_{\ell}\right)}-\mu_{r_{1}, \ldots, r_{\ell}, n, m, k}^{\left(j_{1}, \ldots, j_{\ell}\right)} & =-\frac{j_{\ell}}{a c \gamma_{r_{\ell}}} E\left\{\Psi _ { j _ { \ell } , \ldots , j _ { 1 } } \left(X_{r_{\ell}, n, m, k}, \ldots,\right.\right. \\
& \left.\left.X_{r_{1}, n, m, k}\right)\right\}+\xi \frac{Q-1}{Q} \Phi_{r_{1}, \ldots, r_{\ell}, n-1, m, k+m}^{\left(j_{1}, \ldots, j_{\ell}\right)} .
\end{aligned}
$$




\section{Applications}

In this section, two applications of the previous results are introduced, the first one is the characterizations of members of the class based on recurrence relations for moments of GOS, OOS and k-RVs for doubly truncated modified Weibull (Weibull, Extreme-value, exponential), doubly truncated inverse Weibull distributions and doubly truncated three-parameters Burr type XII (Lomax and Rayleigh). In the second application we will find the Tables of single and product moments of OOS arising from doubly truncated Lomax distribution.

1. Doubly truncated new modified Weibull distribution

$a=1, b=0, c=1$ and $h(x)=\exp \left\{-\theta x^{p} \exp (\lambda x)\right\}$, then the recurrence relations (17), (25) and (27), reduce respectively, to

$$
\begin{gathered}
\mu_{r_{1}, \ldots, r_{\ell}, n, m, k}^{\left(j_{1}, \ldots, j_{\ell}\right)}=\mu_{r_{1}, \ldots, r_{\ell}^{*}, n, m, k}^{\left(j_{1}, \ldots, j_{\ell}\right)}+\frac{j_{\ell}}{\gamma_{r_{\ell}} \theta} E\left\{\frac{X_{r_{1}, \ldots, r_{\ell}, n, m, k}^{j_{\ell}-p} \exp \left(-\lambda X_{r_{1}, \ldots, r_{\ell}, n, m, k}\right)}{p+\lambda X_{r_{1}, \ldots, r_{l}, n, m, k}}\right. \\
\left.\prod_{i=1}^{\ell-1} X_{r_{i}, n, m, k}^{j_{i}}\right\}+\xi Q_{2} \Phi_{r_{1}, \ldots, r_{\ell}, n-1, m, k+m}, \\
\mu_{r, n, m, k}^{(j)}=\mu_{r-1, n, m, k}^{(j)}+\frac{j}{\gamma_{r} \theta} E\left\{\frac{X_{r, n, m, k}^{j-p} \exp \left(-\lambda X_{r, n, m, k}\right)}{p+\lambda X_{r, n, m, k}}\right\}+\xi Q_{2} \Phi_{r, n-1, m, k+m}
\end{gathered}
$$

and

$$
\begin{aligned}
\mu_{r, s, n, m, k}^{(e, \varepsilon)}= & \mu_{r, s-1, n, m, k}^{(e, \varepsilon)}+\frac{\varepsilon}{\gamma_{s} \theta} E\left\{\frac{X_{r, n, m, k}^{e} X_{s, n, m, k}^{\varepsilon-p} \exp \left(-\lambda X_{s, n, m, k}\right)}{p+\lambda X_{s, n, m, k}}\right\} \\
& +\xi Q_{2} \Phi_{r, s, n-1, m, k+m}
\end{aligned}
$$

The recurrence relations (21), (28) and (29), reduce to

$$
\begin{aligned}
\theta\left(n-r_{\ell}+1\right) \mu_{r_{1}, \ldots, r_{\ell}: n}^{\left(j_{1}, \ldots, j_{\ell}\right)}= & \theta\left(n-r_{\ell}+1\right) \mu_{r_{1}, \ldots, r_{\ell}^{*}: n}^{\left(j_{1}, \ldots, j_{\ell}\right)} \\
& +j_{\ell} E\left\{\frac{X_{r_{1}, \ldots, r_{\ell}: n}^{j_{\ell}-p} \exp \left(-\lambda X_{r_{1}, \ldots, r_{\ell}: n}\right)}{p+\lambda X_{r_{1}, \ldots, r_{\ell}: n}} \prod_{i=1}^{\ell-1} X_{r_{i}: n}^{j_{i}}\right\} \\
& +\frac{n}{\left(n-r_{\ell}+1\right)} Q_{2} \Phi_{r_{1}, \ldots, r_{\ell}, n-1},
\end{aligned}
$$


$\left.\left.\mu_{r: n}^{(j)}=\mu_{r-1: n}^{(j)}+\frac{j}{\theta(n-r+1)} E\right\} \frac{X_{r: n}^{j-p} \exp \left(-\lambda X_{r: n}\right)}{p+\lambda X_{r: n}}\right\}+\frac{n \theta}{\theta(n-r+1)} Q_{2} \Phi_{r: n-1}$,

and

$$
\begin{aligned}
\mu_{r, s: n}^{(e, \varepsilon)}= & \mu_{r, s-1: n}^{(e, \varepsilon)}+\frac{\varepsilon}{\theta(n-s+1)} E\left\{\frac{X_{r: n}^{e} x_{s: n}^{\varepsilon-p} \exp \left(-\lambda x_{s: n}\right)}{p+\lambda x_{s: n}}\right\} \\
& +\frac{n \theta}{\theta(n-s+1)} Q_{2} \Phi_{r, s: n-1} .
\end{aligned}
$$

The recurrence relations (23), (30) and (31), reduce to

$$
\begin{gathered}
\mu_{\left(r_{1}, \ldots, r_{l}, k\right)}^{\left(j_{1}, \ldots, j_{\ell}\right)}=\mu_{\left(r_{1}, \ldots, r_{\ell} ; k\right)}^{\left(j_{1}, \ldots, j_{\ell}\right)}+\frac{j_{\ell}}{\theta k} E\left[\frac{x_{\left(r_{1}, \ldots, r_{\ell} ; k\right)}^{j_{\ell}-p} \exp \left\{-\lambda X_{\left(r_{1}, \ldots, r_{\ell} ; k\right)}\right\}}{\left(p+\lambda X_{\left(r_{1}, \ldots, r_{\ell} ; k\right)}\right)} \prod_{i=1}^{\ell-1} X_{\left(r_{i} ; k\right)}^{j_{i}}\right] \\
+Q_{2}\left(1-k^{-1}\right)^{1-r_{\ell}} \Phi_{r_{1}, \ldots, r_{\ell}, k-1}, \\
\mu_{(r, k)}^{(j)}=\mu_{(r-1, k)}^{(j)}+\frac{j}{\theta k} E\left[\frac{X_{(r ; k)}^{j-p} \exp \left\{-\lambda X_{(r ; k)}\right\}}{p+\lambda X_{(r ; k)}}\right]+Q_{2}\left(1-k^{-1}\right)^{1-r} \Phi_{r, k-1},
\end{gathered}
$$

and

$$
\begin{aligned}
\theta k \mu_{(r, s, k)}^{(e, \varepsilon)}= & \theta k \mu_{(r, s-1, k)}^{(e, \varepsilon)}+\varepsilon E\left[\frac{X_{(r, s ; k)}^{e} X_{(r, s ; k)}^{\varepsilon-p} \exp \left\{-\lambda X_{(r, s ; k)}\right\}}{p+\lambda X_{(r, s ; k)}}\right] \\
& +\theta k Q_{2}\left(1-k^{-1}\right)^{1-r} \Phi_{r, s, k-1} .
\end{aligned}
$$

I) If we put $\lambda=0$, we obtain the recurrence relations for Weibull distribution.

II) If we put $p=0$, we obtain the recurrence relations for Extreme-value distribution.

\section{Doubly truncated inverse Weibull distribution}

$a=-1, b=1, c=1$ and $h(x)=\exp \left[-\theta x^{-p}\right]$, then the recurrence relations (17), (25) and (27), reduce respectively, to

$\mu_{r_{1}, \ldots, r_{\ell}, n, m, k}^{\left(j_{1}, \ldots, j_{\ell}\right)}-\mu_{r_{1}, \ldots, r_{\ell}^{*}, n, m, k}^{\left(j_{1}, \ldots, j_{\ell}\right)}=\frac{j_{\ell}}{\gamma_{r_{\ell}} \theta p} E\left[X_{r_{1}, \ldots, r_{\ell}, n, m, k}^{j_{\ell}+p}\left\{\exp \left(\theta X_{r_{1}, \ldots, r_{\ell}, n, m, k}^{-p}\right)-1\right\}\right.$ 


$$
\begin{gathered}
\left.\prod_{i=1}^{\ell-1} X_{r_{i}, n, m, k}^{j_{i}}\right]+\xi Q_{2} \Phi_{r_{1}, \ldots, r_{\ell}, n-1, m, k+m}, \\
\mu_{r, n, m, k}^{(j)}-\mu_{r-1, n, m, k}^{(j)}= \\
\frac{j}{\gamma_{r} \theta p} E\left[X_{r, n, m, k}^{j+p}\left\{\exp \left(\theta X_{r, n, m, k}^{-p}\right)-1\right\}\right] \\
+\xi Q_{2} \Phi_{r, n-1, m, k+m},
\end{gathered}
$$

and

$$
\begin{aligned}
\mu_{r, s, n, m, k}^{(e, \varepsilon)}-\mu_{r, s-1, n, m, k}^{(e, \varepsilon)}= & \frac{\varepsilon}{\gamma_{s} \theta p} E\left[X_{r, n, m, k}^{e} X_{s, n, m, k}^{\varepsilon+p}\left\{\exp \left(\theta X_{s, n, m, k}^{-p}\right)-1\right\}\right] \\
& +\xi Q_{2} \Phi_{r, s, n-1, m, k+m} .
\end{aligned}
$$

The recurrence relations (21), (28) and (29), reduce to

$$
\begin{aligned}
\mu_{r_{1}, \ldots, r_{\ell}: n}^{\left(j_{1}, \ldots, j_{\ell}\right)}-\mu_{r_{1}, \ldots, r_{\ell}: n}^{\left(j_{1}, \ldots, j_{\ell}\right)}= & \frac{j_{\ell}}{\theta p\left(n-r_{\ell}+1\right)} E\left[X_{r_{1}, \ldots, r_{\ell}: n}^{j_{\ell}+p}\left\{\exp \left(\theta X_{r_{1}, \ldots, r_{\ell}: n}^{-p}\right)-1\right\}\right. \\
& \left.\prod_{i=1}^{\ell-1} X_{r_{i}: n}^{j_{i}}\right]+\frac{n}{n-r_{\ell}+1} Q_{2} \Phi_{r_{1}, \ldots, r_{\ell}, n-1} \\
\mu_{r: n}^{(j)}-\mu_{r-1: n}^{(j)}= & \frac{j}{\theta p(n-r+1)} E\left[X_{r: n}^{j+p}\left\{\exp \left(\theta X_{r: n}^{-p}\right)-1\right\}\right] \\
& +\frac{n}{(n-r+1)} Q_{2} \Phi_{r: n-1}
\end{aligned}
$$

and

$$
\begin{aligned}
\mu_{r, s: n}^{(e, \varepsilon)}= & \mu_{r, s-1: n}^{(e, \varepsilon)}+\frac{\varepsilon}{\theta p(n-s+1)} E\left[X_{r: n}^{e} x_{s: n}^{\varepsilon+p}\left\{\exp \left(X_{s: n}^{-p}\right)-1\right\}\right] \\
& +\frac{n}{n-s+1} Q_{2} \Phi_{r, s: n-1} .
\end{aligned}
$$

The recurrence relations (23), (30) and (31), reduce to

$$
\begin{aligned}
\mu_{\left(r_{1}, \ldots, r_{l}, k\right)}^{\left(j_{1}, \ldots, j_{\ell}\right)}= & \mu_{\left(r_{1}, \ldots, r_{\ell}^{*} ; k\right)}^{\left(j_{1}, \ldots, j_{\ell}\right)}+\frac{j_{\ell}}{\theta p k} E\left[x_{\left(r_{1}, \ldots, r_{\ell} ; k\right)}^{j_{\ell}+p}\left[\exp \left\{\theta X_{\left(r_{1}, \ldots, r_{\ell} ; k\right)}^{-p}\right\}-1\right] \prod_{i=1}^{\ell-1} X_{\left(r_{i} ; k\right)}^{j_{i}}\right] \\
& +Q_{2}\left(1-k^{-1}\right)^{1-r_{\ell}} \Phi_{r_{1}, \ldots, r_{\ell}, k-1},
\end{aligned}
$$




$$
\begin{aligned}
\mu_{(r, k)}^{(j)}= & \mu_{(r-1, k)}^{(j)}+\frac{j}{\theta p k} E\left[X_{(r ; k)}^{j+p}\left[\exp \left\{\theta X_{(r ; k)}^{-p}\right\}-1\right]\right] \\
& +Q_{2}\left(1-k^{-1}\right)^{1-r} \Phi_{r, k-1},
\end{aligned}
$$

and

$$
\begin{aligned}
\mu_{(r, s, k)}^{(e, \varepsilon)}= & \mu_{(r, s-1, k)}^{(e, \varepsilon)}+\frac{\varepsilon}{\theta p k} E\left[X_{(r, s ; k)}^{e} X_{(r, s ; k)}^{\varepsilon+p}\left[\exp \left\{\theta X_{(r, s ; k)}^{-p}\right\}-1\right]\right] \\
& +Q_{2}\left(1-k^{-1}\right)^{1-r} \Phi_{r, s, k-1}
\end{aligned}
$$

3. Doubly truncated Burr Type XII distribution.

$a=\frac{1}{\sigma}, b=1, c=-\theta$ and $h(x)=x^{\eta}$, then the recurrence relations (17), (25) and (27), reduce respectively, to

$$
\begin{aligned}
&\left(\eta \gamma_{r_{\ell}} \theta-j_{\ell}\right) \mu_{r_{1}, \ldots, r_{\ell}, n, m, k}^{\left(j_{1}, \ldots, j_{\ell}\right)}-\eta \gamma_{r_{\ell}} \theta \mu_{r_{1}, \ldots, r_{\ell}^{*}, n, m, k}^{\left(j_{1}, \ldots, j_{\ell}\right)}= \sigma j_{\ell} \mu_{r_{1}, \ldots, r_{\ell}, n, m, k}^{\left(j_{1}, \ldots, j_{\ell-\eta)}\right.} \\
&+\eta \theta \gamma_{r_{\ell}} \xi Q_{2} \Phi_{r_{1}, \ldots, r_{\ell}, n-1, m,}^{\left(j_{1}, \ldots, j_{\ell}\right)} \\
&\left(\eta \gamma_{r} \theta-j\right) \mu_{r, n, m, k}^{(j)}-\eta \gamma_{r} \theta \mu_{r-1, n, m, k}^{(j)}=\sigma j \mu_{r, n, m, k}^{(j-\eta)}+\eta \theta \xi \gamma_{r} Q_{2} \Phi_{r, n-1, m, k+m}^{(j)}
\end{aligned}
$$

and

$$
\begin{aligned}
\left(\eta \theta \gamma_{s}-\varepsilon\right) \mu_{r, s, n, m, k}^{(e, \varepsilon)}= & \eta \gamma_{s} \theta \mu_{r, s-1, n, m, k}^{(e, \varepsilon)}+\sigma \varepsilon \mu_{r, s, n, m, k}^{(e, \varepsilon-\eta)} \\
& +\eta \theta \gamma_{s} \xi Q_{2} \Phi_{r, s, n-1, m, k+m}^{(e, \varepsilon)}
\end{aligned}
$$

The recurrence relations (21), (28) and (29), reduce respectively, to

$$
\begin{aligned}
\left\{\eta \theta\left(n-r_{\ell}+1\right)-j_{\ell}\right\} \mu_{r_{1}, \ldots, r_{\ell}: n}^{\left(j_{1}, \ldots, j_{\ell}\right)}-\eta \theta\left(n-r_{\ell}+1\right) \mu_{r_{1}, \ldots, r_{\ell}^{*}: n}^{\left(j_{1}, \ldots, j_{\ell}\right)} & =\sigma j_{\ell} \mu_{r_{1}, \ldots, r_{\ell}: n}^{\left(j_{1}, \ldots, j_{\ell}-\eta\right)} \\
& +\eta \theta n Q_{2} \Phi_{r_{1}, \ldots, r_{\ell}: n-1}^{\left(j_{1}, \ldots, j_{\ell}\right)}
\end{aligned}
$$

$$
\{\eta \theta(n-r+1)-j\} \mu_{r: n}^{(j)}-\eta \theta(n-r+1) \mu_{r-1: n}^{(j)}=\sigma j \mu_{r: n}^{(j-\eta)}+\eta \theta n Q_{2} \Phi_{r: n-1}^{(j)}
$$

and

$$
\begin{aligned}
\{\eta \theta(n-s+1)-\varepsilon\}) \mu_{r, s: n}^{(e, \varepsilon)}-\eta \theta(n-s+1) \mu_{r, s-1: n}^{(e, \varepsilon)} & =\sigma \varepsilon \mu_{r, s: n}^{(e, \varepsilon-\eta)} \\
& +\eta \theta n Q_{2} \Phi_{r, s: n-1}^{(e, \varepsilon)}
\end{aligned}
$$


The recurrence relations (23), (30) and (31), reduce respectively, to

$$
\begin{aligned}
&\left(\eta \theta k-j_{\ell}\right) \mu_{\left(r_{1}, \ldots, r_{\ell}, k\right)}^{\left(j_{1}, \ldots, j_{\ell}\right)}-\eta \theta k \mu_{\left(r_{1}, \ldots, r_{\ell}^{*} ; k\right)}^{\left(j_{1}, \ldots, j_{\ell}\right)}= \sigma j_{\ell} \mu_{\left(r_{1}, \ldots, r_{\ell}, k\right)}^{\left(j_{1}, \ldots, j_{\ell}-\eta\right)}+\eta \theta k Q_{2}\left(1-k^{-1}\right)^{1-r_{\ell}} \\
& \times \Phi_{\left(r_{1}, \ldots, r_{\ell}, k-1\right)}^{\left(j_{1}, \ldots, j_{\ell}\right)} \\
&(\eta \theta k-j) \mu_{(r, k)}^{(j)}-\eta \theta k \mu_{(r-1, k)}^{(j)}=\sigma j \mu_{(r, k)}^{(j-\eta)}+\eta \theta k Q_{2}\left(1-k^{-1}\right)^{1-r} \Phi_{(r, k-1)}^{(j)}
\end{aligned}
$$

and

$$
\begin{aligned}
(\eta \theta k-\varepsilon) \mu_{(r, s, k)}^{(e, \varepsilon)}-\eta \theta k \mu_{(r, s-1, k)}^{(e, \varepsilon)}= & \sigma \varepsilon \mu_{(r, s, k)}^{(e, \varepsilon-\eta)}+\eta \theta k Q_{2}\left(1-k^{-1}\right)^{1-r} \\
& \times \Phi_{(r, s, k-1)}^{(e, \varepsilon)} .
\end{aligned}
$$

When $\eta=1$ in (54), (55), (56), (57), (58), (59), (60), (61) and (62), the recurrence relations for the Lomax distribution are obtained.

\subsection{Another Application}

In this application we compute numerically the single moments of OOS from doubly truncated Lomax distribution.

The recurrence relations of single and product moments of OOS from doubly truncated Lomax distribution can be obtained as

$$
\begin{gathered}
{[\theta(n-r+1)-1] \mu_{r: n}=\theta(n-r+1) \mu_{r-1: n}+\theta n Q_{2}\left[\mu_{r: n-1}-\mu_{r-1: n-1}\right]+\sigma(63)} \\
{[\theta(n-s+1)-1] \mu_{r, s: n}=\theta(n-s+1) \mu_{r, s-1: n}+\theta n Q_{2}\left[\mu_{r, s: n-1}-\mu_{r, s-1: n-1}\right]+\sigma \mu_{r: n}}
\end{gathered}
$$

Suppose that : $P=0.25, Q=0.75$ and then $Q_{2}=(Q-1) /(Q-P)=-0.5$. We consider the following three cases of the parameter values:

i) If $\theta=3$ and $\sigma=1$, then the $c d f$ of Lomax distribution is $F(x)=1$ $(1+x)^{-3}$, so we can obtain $P_{1}$ and $Q_{1}$ from $P=F\left(P_{1}\right)$ and $Q=F\left(Q_{1}\right)$ it is:

$$
P_{1}=0.1006 \text { and } Q_{1}=0.5874
$$

ii) If $\theta=3$ and $\sigma=2$, then the $c d f$ of Lomax distribution is $F(x)=$ $1-\left(1+\frac{x}{2}\right)^{-3}$, so we can find $P_{1}$ and $Q_{1}$ as:

$$
P_{1}=0.2012 \text { and } Q_{1}=1.1748 .
$$


iii) If $\theta=3$ and $\sigma=3$, then the $c d f$ of Lomax distribution is $F(x)=$ $1-\left(1+\frac{x}{3}\right)^{-3}$, so we can obtain $P_{1}$ and $Q_{1}$ as:

$$
P_{1}=0.301927 \text { and } Q_{1}=1.7622 \text {. }
$$

and we will use the relations.

$$
E\left(X_{0: n}^{j}\right)=P_{1}^{j}, \quad n \geqslant 0 \text { and } E\left(X_{n: n-1}^{j}\right)=Q_{1}^{j}, \quad n \geqslant 1 .
$$

(see Khan and Khan, 1987).

Table 1. The single moments of OOS from doubly truncated Lomax distribution.

\begin{tabular}{ccccc}
\hline \hline$r$ & $n$ & \multicolumn{3}{c}{$\mu_{r: n}$} \\
\cline { 3 - 5 } & & $\theta=3, \sigma=1$ & $\theta=3, \sigma=2$ & $\theta=3, \sigma=3$ \\
\hline 1 & 1 & 0.2859 & 0.5718 & 0.8576 \\
1 & 2 & 0.2096 & 0.4192 & 0.6288 \\
2 & 2 & 0.3622 & 0.7243 & 1.0865 \\
1 & 3 & 0.1769 & 0.3538 & 0.5307 \\
2 & 3 & 0.2750 & 0.5500 & 0.8250 \\
3 & 3 & 0.4057 & 0.8115 & 1.2172 \\
1 & 4 & 0.1591 & 0.3182 & 0.4772 \\
2 & 4 & 0.2304 & 0.4608 & 0.6912 \\
3 & 4 & 0.3196 & 0.6392 & 0.9588 \\
4 & 4 & 0.4344 & 0.8689 & 1.3033 \\
1 & 5 & 0.1479 & 0.2959 & 0.4438 \\
2 & 5 & 0.2037 & 0.4073 & 0.6110 \\
3 & 5 & 0.2705 & 0.5410 & 0.8115 \\
4 & 5 & 0.3524 & 0.7047 & 1.0570 \\
5 & 5 & 0.4549 & 0.9099 & 1.3649 \\
\hline
\end{tabular}

Table 2. The product moments of OOS from doubly truncated Lomax distribution.

\begin{tabular}{cccccc}
\hline \hline$r$ & $s$ & $n$ & \multicolumn{3}{c}{$\mu_{r, s: n}$} \\
\cline { 4 - 6 } & & & $\theta=3, \sigma=1$ & $\theta=3, \sigma=2$ & $\theta=3, \sigma=3$ \\
\hline 1 & 2 & 2 & 0.0817 & 0.3269 & 0.7356 \\
1 & 2 & 3 & 0.0527 & 0.2108 & 0.4743 \\
1 & 3 & 3 & 0.0743 & 0.2975 & 0.6694 \\
2 & 3 & 3 & 0.1180 & 0.4724 & 1.0631 \\
1 & 2 & 4 & 0.0393 & 0.1575 & 0.3544 \\
1 & 3 & 4 & 0.0530 & 0.2122 & 0.4775 \\
1 & 4 & 4 & 0.0705 & 0.2820 & 0.6345 \\
2 & 3 & 4 & 0.0790 & 0.3159 & 0.7109 \\
2 & 4 & 4 & 0.1034 & 0.4137 & 0.9309 \\
3 & 4 & 4 & 0.1449 & 0.5801 & 1.3052 \\
\hline
\end{tabular}


Note: It should be noted from Tables 1 and 2 that

i) The values of different moments are decreasing with increasing the sample size $n$.

ii) The following relation

$$
\sum_{i=1}^{n} \mu_{i: n}=n E(X)=n \mu_{1: 1}
$$

is satisfied for all values of single moments in Table 1.

iii) The following relation

$$
\sum_{i=1}^{n-1} \sum_{j=i+1}^{n} \mu_{i, j: n}=\sum_{i=1}^{n-1} \sum_{j=i+1}^{n} E\left(X_{i: n} X_{j: n}\right)=\frac{n(n-1)}{2} \mu_{1: 1}^{2}
$$

is holds for all values of product moments in Table 2 .

\section{References}

Ahmad, A.A., (2001). Moments of order statistics from doubly truncated continuous distribution and characterizations. Statistics, 35, 479-494.

Ahmad, A.A. (2007). Relations for single and product moments of generalized order statistics from doubly truncated Burr type XII distribution. J. Egypt. Math. Soc., 15.

Ahmad, A.A. (2008). Single and product moments of generalized order statistics from linear exponential distribution. Commun. Statist. Theory Meth., 37, 1-11.

Ahmad, A.A. and Abu-Shal, T.A. (2006). Recurrence relations for moment generating functions of nonadjacent generalized order statistics based on a class of doubly truncated distributions. Journal of Statistical Theory and Applications, 6, 174-189.

Ahmad, A.A. and Fawzy, M. (2003). Recurrence relations for single moments of generalized order statistics from doubly truncated distributions and its characterizations. J. Statist. Plann. and Inference 177, 241-249.

Ahsanullah, M., (1995), Record Statistics (Commack NY: Nova Science Publisher, Inc.).

Ahsanullah, M. (2000). Generalized order statistics from exponential distribution. J. Statist. Plann. and Inference, 85, 85-91. 
AL-Hussaini, E.K., Ahmad, A.A. and AL-Kashif, M.A. (2005). Recurrence relations for moment and conditional moment generating functions of generalized order statistics. Metika, 61, 199-220.

AL-Hussaini, E.K., Ahmad, A.A. and EL-Boghdady, H.H., (2004) Recurrence relations for moments generating function of order statistics. Meton LXII, 1, 85-99.

Arnold, B.C., Balakrishnan, N. and Nagaraja, H.N., (1992). A First Course in Order Statistics. Wiley, NewYork.

Athar, H. and Islam, H.M. (2004). Recurrence relations for single and product moments of generalized order statistics from a general class of distributions. Metron LXII, 3, 327-337.

Balakrishnan, N. and Ahsanullah, M. (1995). Relations for single and product moments of record values from exponential distribution. Commun. Statist. Theory Meth., 23, 2841-2852.

Balakrishnan, N. and Asgharzadeh, (2005), A. Inference for the scaled half-logistic distribution based on progressively type-II censored samples. Comm. Statist. Theory Methods, 34, $73-87$.

Balakrishnan, N., Malik, H.J., and Ahmed, S.E., (1988). Recurrence relations and identities for moments of order statistics, I: Specific continuous distributions. Commun. Statistics. Theory Methods, 17, 3623-2655.

Balakrishnan, N. and Sultan, K.S. (1998). Recurrence relations and identities for moments of order statistics. Order statistics: theory and methods, 149-228, Handbook of Statist., 16, North-Holland, Amsterdam, 62-02 (62G30).

Cramer, E. and Kamps, U. (1996). Sequential order statistics and k-out-of-n systems with sequantially adjusted failure rates. Annals of Institute of Statistical Mathematics, 48, 535-549.

Cramer, E. and Kamps, U. (2000). Relations for expectations of functions of generalized order statistics. J. Statist. Plann. and Inference, 89, 79-89.

Min Xie, C.D.L. and Murthy, D.N.P. (2003). A Modified Weibull Distribution. IEEE Trans. Rel., 52, 33-37.

David, H.A., (1981). Order Statistics. (2nd edn), Wiley, NewYork.

Habibullah, M. and Ahsanullah, M., (2000). Estimation of parameters of a Pareto distribution by generalized order statistics. Commun. Statist.Theory Meth., 29, 1597-1609.

Hwang, T.Y. and Lin, G.D. (1984). Extension of Müntz-Szàsz theorem and applications. Analysis, 4, 143-160.

Kamps, U. (1995a). A Concept of Generalized Order Statistics. Teubner, Stuttgart. 
Khan, A.H. and Khan, I.A. (1987). Moments of order statistics from Burr distribution and characterizations. Metron, 45, 21-29.

Keseling, C. (1999). Conditional distributions of generalized order statistics and some characterizations. Metrika, 49, 27-40.

Mahmoud, M.A.W. and Al-Nagar, H.S. (2006). Generalized order statistics based on doubly truncated general class of distributions. J. Statist. Theory and Appl., 6, 206-219.

Nevzorov, V.B. (1987). Records. Thoery of Probability and Its Applications, 32, 201-228.

Pawlas, P. and Szynal, D. (1999). Recurrence relations for single and product moments of k-th record values from Pareto, Generalized Pareto and Burr distributions. Commun. Statist.Theory Meth., 28, 1699-1709.

Pawlas, P. and Szynal, D. (2001). Recurrence relations for single and product moments from Pareto, Generalized Pareto and Burr distributions. Commun. Statist.Theory Meth., 30, 739746.

Saran, J. and Pandey, A. (2003). Recurrence relations for marginal and joint moment generating functions of generalized order statistics from Lomax distribution. J. Statist. Studies, 23, 39-44.

\section{A. H. Abd Ellah}

Department of Mathematics, Sohag University,

Sohag 82524, Egypt.

email: ahmhamed@hotmail.com

\author{
Abd El-Baset A. Ahmad \\ Department of Mathematics and Statistics, \\ Taif University, \\ Saudi Arabia.
}

Mohammad A. Fawzy

Department of Mathematics,

Sohag University,

Sohag 82524, Egypt. 